\title{
GAIA Neonatal Infections Level of Diagnostic Certainty
}

National Cancer Institute

\section{Source}

National Cancer Institute. GAlA Neonatal Infections Level of Diagnostic Certainty. NCI

Thesaurus. Code C126844.

A classification of maternal and fetal outcomes relating to neonatal infections, developed by the Global Alignment of Immunization safety Assessment in pregnancy, based on the extent to which the diagnosis has been confirmed. 